TRANSACTIONS OF THE

AMERICAN MATHEMATICAL SOCIETY

Volume 356, Number 3, Pages 1025-1043

S 0002-9947(03)03297-5

Article electronically published on August 25, 2003

\title{
EXTENSIONS CONTAINED IN IDEALS
}

\author{
DAN KUCEROVSKY
}

\begin{abstract}
We prove a Weyl-von Neumann type absorption theorem for extensions which are not full, and give a condition for constructing infinite repeats contained in an ideal. We also clear up some questions associated with the purely large criterion for full extensions to be absorbing.
\end{abstract}

\section{INTRODUCTION}

Kasparov [7] showed that $K K^{1}(A, B)$ can be viewed as a group of absorbing extensions. In [6], the authors introduced the purely large condition and proved that extensions are absorbing in Kasparov's sense if and only if they are purely large and full, so that $K K^{1}(A, B)$ can be regarded as a group of essential, full, and purely large extensions.

The purely large condition is an algebraical condition on a subalgebra of a multiplier algebra associated with a given essential extension. An essential extension of $A$ by $B$ is by definition purely large if the extension algebra satisfies the purely large condition as a subalgebra of the multipliers of the canonical ideal, $B$. (See Theorem 11ii) for the exact definition). We recall that the introduction of the absorption property for extensions was originally motivated by the observation that extensions form a semigroup in a natural way - in the presence of a unital copy of $\mathrm{O}_{2}$ - and it then turned out to be desirable to show that extensions which lift to homomorphisms into the multipliers are actually algebraically trivial in the semigroup. Extensions that lift to homomorphisms in this sense are usually called "trivial extensions." With this terminology, we can say that an extension $\tau$ is absorbing if the semigroup sum of $\tau$ with any trivial extension is unitarily equivalent to $\tau$, or, even more briefly, just that an extension is absorbing if trivial extensions are algebraically trivial with respect to the given extension.

In a previous paper [6], we showed the following equivalence of three conditions, which for convenience we state in a somewhat overly restricted form:

Theorem 1. Let $A$ and $B$ be separable $C^{*}$-algebras, with $B$ stable and $A$ unital. Let $0 \longrightarrow B \longrightarrow C \longrightarrow A \longrightarrow 0$ be an essential unital extension with weakly nuclear splitting morphism $s: A \longrightarrow C$. Then the following are equivalent:

i) The extension is absorbing, in the nuclear sense.

Received by the editors July 29, 2002.

2000 Mathematics Subject Classification. Primary 19K35; Secondary 46L85, 46L80.

Key words and phrases. KK-theory, classification of operator algebras, absorbing extensions. This research was supported by the NSERC, under grant \# 228065-00. 
ii) The extension algebra has the purely large property, i.e. the property that, for every $c \in C^{+}$that is not zero in $C / B$, the hereditary subalgebra that it generates, $\overline{c B c}$, contains a stable subalgebra that is full in $B$.

iii) The extension algebra has the approximation property that, for every $c \in C^{+}$ that is not zero in $C / B$ and every positive $b$ in $B$, there is an element $r \in B$ making the norm of $b-r c r^{*}$ arbitrarily small. Moreover, the element $r$ can be assumed to be of norm one if $b$ and $c$ are.

Note that in the above theorem, it is not assumed that $B$ is simple. However, if $B$ is not simple, the algebra $C$ then has to be full with respect to $B$, so to speak, in a very strong sense. In section 3 of this paper we will be interested in weakening the fullness condition in the above theorem, which in turn will require us to weaken the definition of absorption.

Observe that for nonsimple algebras, or even for simple algebras with nonsimple corona, the ideal structure is in general an obstacle for the absorption of one extension by another. For example, consider two extensions $\tau, \pi: A \longrightarrow \mathcal{M}(B) / B$. If the ideal generated by the image of $\pi$ at some point is strictly larger than the ideal generated by the image of $\tau$ at that point, then $[\tau]$ can never absorb $[\pi]$. This obstacle does not arise if $B$ is simple and purely infinite, since then the multipliers have only the canonical ideal, but in the nonsimple case it must be taken into account. In particular, since it is known that in general there exists a trivial full extension, an extension is absorbing only if it is full.

We say that one extension dominates another if the ideal structure is not an obstacle for the first extension to absorb the second. The exact condition is that:

Definition 2. An extension $\hat{\tau}_{1}: A \longrightarrow \mathcal{M}(B)$ dominates $\hat{\tau}_{2}: A \longrightarrow \mathcal{M}(B)$ if the norm-closed ideal generated by $\hat{\tau}_{1}(a)$ contains $\hat{\tau}_{2}(a)$ for each positive element $a \in A^{+}$.

We now introduce two notions of equivalence for extensions. Note that the second of the two is applicable not to a pair of extensions, but to a pair of distinguished liftings.

Definition 3. i) Two extensions $\tau_{1}, \tau_{2}: A \longrightarrow \mathcal{M}(B \otimes \mathcal{K})$ are said to be equivalent if there is a multiplier unitary $U$ making $\tau_{1}(a)$ and $\tau_{2}(a)$ equivalent modulo $B \otimes \mathcal{K}$ for all $a \in A$.

ii) Two extensions $\tau_{1}, \tau_{2}: A \longrightarrow \mathcal{M}(B \otimes \mathcal{K})$ are said to be approximately unitarily equivalent if for every $\epsilon>0$ and every finite set $\mathcal{A} \subset A$ there is a multiplier unitary $U$ such that

(a) $U \tau_{1}(a) U^{*}-\tau_{2}(a)$ has norm less than $\epsilon$ for all $a$ in the given finite set $\mathcal{A}$, and

(b) the unitary $U$ makes $\tau_{1}$ and $\tau_{2}$ equivalent in the sense of the above definition i).

Thus we see that approximate unitary equivalence of extensions is, despite the name, apparently stronger than ordinary (unitary) equivalence of extensions. However, the most generally applicable way of showing that two extensions are equivalent is to apply a theorem of Weyl-von Neumann type, and in fact, such theorems give approximate unitary equivalence of the lifted extensions as a conclusion (for example, this was the case in the proof of Theorem 1). It is, therefore, tempting to speculate that approximate unitary equivalence and ordinary equivalence give 
rise to the same equivalence classes when applied to lifted extensions. Some insight into the relationship between the two types of equivalence is obtained from considering the following construction, which shows us that it is possible to obtain approximate unitary equivalence from ordinary equivalence of extensions if we are willing to pass to suspensions (which, at the level of Kasparov theory, just replaces $K K^{1}$ by $\left.K K^{0}\right)$ :

Given an extension $\tau: A \longrightarrow \mathcal{M}(B \otimes \mathcal{K})$, we can define another extension $\alpha(\tau)$ : $A \longrightarrow \mathcal{M}(S B \otimes \mathcal{K})$ by mapping $(a, t)$ to $t \tau(a)$, where $t$ is the parameter defining the suspension. If $\alpha\left(\tau_{1}\right)$ and $\alpha\left(\tau_{2}\right)$ are unitarily equivalent in the standard sense, then $\tau_{1}$ and $\tau_{2}$ are approximately unitarily equivalent. The converse would not immediately hold without a strict continuity condition on the family of extensions.

A minor variation of this construction establishes the following:

Proposition 4. Let $B$ be a stable algebra having the property that every extension by $S B$ is absorbing in the ideal-related sense. Let $c$ be a positive element of $\mathcal{M}(B)$, and let $h$ be an element of the associated norm-closed multiplier ideal, $\overline{\mathcal{M}(B) c \mathcal{M}(B)}$, that has the same spectrum as $c$ in $\mathcal{M}(B)$. Then there exists $r \in$ $\mathcal{M}(B)$ such that $r f(c) r^{*}=f(h)$ modulo $B$ for all functions $f \in C_{0}\left(\mathbb{R}^{+}\right)$. Moreover, $r$ can be chosen to make $r f(c) r^{*}-f(h)$ have arbitrarily small norm.

Proof. Let $c$ and $h$ be as in the hypothesis. Let $\tilde{c}$ and $\tilde{h}$ be the images of $c$ and $h$ in the suspension $\mathcal{M}(S B)$, under the natural embedding as constant functions. The isospectrality condition lets us map $C^{*}(\tilde{h})$ to $C^{*}(\tilde{c})$, using the Gelfand theorem. Thus we can define

$$
\begin{aligned}
\Psi: C^{*}(\tilde{h}) / S B & \longrightarrow \mathcal{M}(S B) / S B \\
f(\tilde{h}) & \mapsto t f(\tilde{c})
\end{aligned}
$$

and

$$
\begin{aligned}
\Phi: C^{*}(\tilde{h}) / S B & \longrightarrow \mathcal{M}(S B) / S B \\
f(\tilde{h}) & \mapsto t f(\tilde{h}),
\end{aligned}
$$

where $t$ is the parameter defining the suspension. By isospectrality, these nuclear maps are injective, and therefore define essential extensions. Since $\Phi$ is trivial and $\tilde{h}$ is in the ideal generated by $\tilde{c}$, we have that $\Psi$ absorbs $\Phi$. Hence there is a unitary in $\mathcal{M}(B) \otimes C[0,1]$ such that $\Psi \oplus \Phi=U \Psi U^{*}$, where the addition is understood to take place in the semigroup of extensions, as defined by Brown, Douglas, and Fillmore [3]. Next, we cut down by a projection to obtain $\Phi(f)=r \Psi(f) r^{*}$ modulo $S B$, or $\operatorname{tf}(\tilde{h})=\operatorname{trf}(\tilde{c}) r^{*}+\tilde{b}$ for some $\tilde{b} \in C_{0}(0,1) \otimes B$. Evaluating this at some value of $t$ close to 1 , we obtain the claimed norm estimate.

The conclusion of the next corollary is a weaker form of the condition in part iii) of Theorem 1

Corollary 5. Let $B$ be a stable algebra having the property that every extension by $S B$ is absorbing in the ideal-related sense. Let $c$ be a positive element of the multipliers $\mathcal{M}(B)$, with spectrum $[0,\|c\|]$. Then, for all $h$ in the positive cone of the hereditary subalgebra $\overline{c \mathcal{M}(B) c}$, we can approximate $h$ by $r c r^{*}$ for some $r$ in the multipliers. If the norm of $h$ is the same as the norm of $c$, we can take the norm of $r$ to be approximately equal to 1. 
Proof. Let us suppose that the norm of the given $c$ and $h$ are both equal to 1 . Since $h$ has a square root in $\overline{c \mathcal{M}(B) c}$, we can use Cohen's theorem [4] to factorize $h^{1 / 2}$ as $f(c) h^{\prime} f(c)$ for some $h^{\prime}$ with norm approximately equal to one, and some $f(c)$ with norm equal to one. Noting that $c$ and $f(c)^{2}$ are isospectral, we can find an $r$ of norm approximately equal to one, such that $f(c)^{2}$ is approximately equal to $r c r^{*}$. Thus, $h$ is approximated by $f(c) h^{\prime} r c r^{*} h^{\prime} f(c)$.

Given the observations made above, it seems reasonable to take approximate unitary equivalence of extensions to be the main form of equivalence that we consider. Naturally, this forces us to work with lifted extensions, that is, to consider extensions as being completely positive, nuclear, unital maps into a multiplier algebra. In this context, a trivial extension is one that happens to be a homomorphism.

We formalize the situation we are interested by defining absorption with respect to ideals:

Definition 6. An extension $\hat{\tau}: A \longrightarrow \mathcal{M}(B)$ is absorbing with respect to ideals if its semigroup sum with a trivial extension that it dominates is approximately unitarily equivalent to the original extension $\hat{\tau}$.

Corollary $[5$ motivates the following definition, where the subalgebra $\overline{c \mathcal{M}(B) c}$ appearing in the corollary has been restricted to its intersection with the canonical ideal $B$ :

Definition 7. Let $C$ be a subalgebra of the multipliers $\mathcal{M}(B)$. We say that the algebra is factorizable with respect to $B$ if for every positive $c$ in $C$ having the property that the spectrum of $c$ is $[0,\|c\|]$, we can approximate positive elements $h$ of $\overline{c B c}$ by $r c r^{*}$ for some $r$ in $B$. If the norm of $h$ is the same as the norm of $c$, we can take the norm of $r$ to be approximately equal to 1 .

We shall see that homomorphisms from a factorizable algebra to a factorizable algebra have a kind of rigidity property with respect to ideals that we shall exploit in proving a Weyl-von Neumann type theorem.

Lastly, let us note that the factorizability condition is vacuously satisfied if there are no elements $c \in C$ such that $C^{*}(c)$ has connected spectrum in the corona! In order to avoid this trivial case, we shall therefore place a minor technical condition, related to Zhang's dichotomy [14], on the algebras we work with:

Definition 8. Let $C$ be a unital subalgebra of the multipliers $\mathcal{M}(B)$. We say that $C / B$ has property (CS) if every hereditary kernel (of a pure state) in the quotient $C / B$ contains a strictly positive element $h$ with connected spectrum $\operatorname{sp}_{C / B} h$.

In terms of the application to extensions, the $C S$ condition is a condition on the quotient algebra that will guarantee us the existence of enough elements with good properties.

\section{INFINITE REPEATS OF EXTENSIONS}

So far, we have only dealt with finite sums of extensions. In applications, we very often need to work with infinite sums of extensions. For example, if $\tau$ is a trivial extension that is absorbing in some sense, we would expect that $\tau$ would absorb its infinite repeat.

By an infinite repeat, we mean an infinite sum, $\tau \oplus \tau \oplus \tau \oplus \cdots$, where $\tau$ is a trivial, essential, and nuclear extension of separable $C^{*}$-algebras. One must ask how 
exactly the limit is defined when taking an infinite sum of extensions: defining a $*$ homomorphism $\delta_{\infty}: \mathcal{M}(B \otimes \mathcal{K}) \longrightarrow \mathcal{M}(B \otimes \mathcal{K})$ by $c \mapsto \sum_{1}^{\infty} v_{i} c v_{i}^{*}$ (with convergence in the strict topology), where $\left(v_{i}\right)$ is a set of generators for a copy of $O_{\infty}$ in the multipliers, one has that this homomorphism, when applied to an extension, is the infinite repeat of that extension.

It turns out that the factorization condition is not sufficient to insure that trivial extensions absorb their infinite repeats, and we need a second, more directly idealrelated condition, as well. The relevant condition is tentatively called the local fullness property:

Definition 9. We say that an extension is locally full if the extension algebra $C$, regarded as a subalgebra of the multipliers $\mathcal{M}(B)$, has the property that every positive element, $c \in C^{+}$, is full in $\frac{\mathcal{M}(\overline{B c B)}}{\overline{B c B}}$.

For brevity, we sometimes refer to $\frac{\mathcal{M}(\overline{B c B})}{\overline{B c B}}$ as the local corona. The local fullness condition will be studied in greater detail later. We point out that not every extension is locally full, since in general there exist multiplier elements $c \in \mathcal{M}(B)^{+}$ which are full in the canonical ideal but are not full in the multipliers (as can be seen in the case of a stabilized UHF algebra [13]).

We begin with some results on hereditary subalgebras.

Lemma 10. Let $B$ be stable and $\sigma$-unital. A hereditary subalgebra, $\overline{\ell B \ell}$, generated by a positive element $\ell$ of the multipliers $\mathcal{M}(B)$ is unitarily equivalent to a hereditary subalgebra generated by a multiplier projection.

Proof. Let $\tilde{B}$ denote the unitization of $B$. The closed right ideal $E:=\overline{\ell B}$ is a countably generated Hilbert $\tilde{B}$-module with inner product $\langle a, b\rangle:=a^{*} b$. Thus, by the Kasparov stabilization theorem [7, there is a unitary $U$ in $\mathcal{L}\left(E \oplus \mathcal{H}_{\tilde{B}}, \mathcal{H}_{\tilde{B}}\right)$ implementing an isomorphism of $E \oplus \mathcal{H}_{\tilde{B}}$ and $\mathcal{H}_{\tilde{B}}$.

Let $P$ be the projection of $E \oplus \mathcal{H}_{\tilde{B}}$ onto the first factor, $E$. The projection $T:=U P U^{*} \in \mathcal{L}\left(\mathcal{H}_{\tilde{B}}\right)$ has image isomorphic (by a unitary equivalence) to $E$, and thus by the definition of the compact operators on a Hilbert module,

$$
\mathcal{K}\left(T \mathcal{H}_{\tilde{B}}\right) \cong \mathcal{K}(\overline{\ell B})
$$

or $T \mathcal{K}\left(\mathcal{H}_{\tilde{B}}\right) T \cong \overline{\ell B \ell}$. Since $B$ is stable, its unitization is just $\mathbb{C} \oplus B$, and $\mathcal{L}\left(\mathcal{H}_{\tilde{B}}\right)$ can be identified with $\mathcal{M}(K \oplus B)$. Thus we see that

$$
0 \oplus \overline{\ell B \ell}=T(K \oplus B) T
$$

(where $K$ denotes the usual compact operators on Hilbert space) for a projection $T$ in $\mathcal{M}(K \oplus B)$. However, since the kernel of $T$ contains $K$, we actually have $T:=0 \oplus T^{\prime}$, where $T^{\prime}$ is in $\mathcal{M}(B)$.

We thank George Elliott for suggesting the next lemma:

Lemma 11. Let $P$ be a projection in some multiplier algebra $\mathcal{M}(B)$. Then

$$
P \mathcal{M}(B) P=\mathcal{M}(P B P) .
$$

Proof. It is sufficient to prove that $\mathcal{M}(P B P)$ is contained in $\mathcal{M}(B)$. An element $v$ of $\mathcal{M}(P B P)$ is in the double dual of $P B P$ and hence is in the double dual of $B$, so the product $b v$, where $b$ is in $B$, is well defined, and we just have to show that it gives an element of $B$, rather than $B^{* *}$. 
Decomposing $b$ as an operator matrix with respect to $P$ :

$$
\left(\begin{array}{cc}
P b P & P b(1-P) \\
(1-P) b P & (1-P) b(1-P)
\end{array}\right),
$$

which is approximately equal to (by means of an approximate unit for $B$ ) the operator

$$
\left(\begin{array}{cc}
P b P & P b_{0} P b(1-P) \\
(1-P) b P b_{0} P & (1-P) b(1-P)
\end{array}\right),
$$

where we have that the product $v P b_{0} P$ is in $P B P$, and the product $v(1-P)$ is zero. Finally, $v P b P$ is also in $P B P$, and hence the product of the above matrix decomposition with $v$ is in fact an element of $B$.

Now, let us see in greater detail how the local fullness condition is related to the property that a trivial extension $\tau$ is approximately unitarily equivalent to its infinite repeat $\delta_{\infty}(\tau(c))=: \tau(c) \oplus \tau(c) \oplus \tau(c) \oplus \cdots$.

We first note that there is a connection with the notion of purely large subalgebras introduced in [6] (the definition is repeated in part ii) of Theorem [1):

Proposition 12. Let $B$ be stable and $\sigma$-unital. If $c$ is a positive element of the multipliers of $B$ that is unitarily equivalent to $\delta_{\infty}(c)$ modulo $B$, then $\overline{\delta_{\infty}(c) B \delta_{\infty}(c)}$ is stable and full in $\overline{B c B}$.

Proof. Let $\left(v_{i}\right)$ be the isometries defining the map $\delta_{\infty}$. Since, for any $b_{1}, b_{2}$ in $B$, the series

$$
\sum b_{1} v_{j} c v_{j}^{*} b_{2}
$$

converges in norm, we see that the ideal generated by $\delta_{\infty}(c)$ in $B$ is contained in the ideal generated by $c$. Evidently, the reverse inclusion also holds, and thus $c$ and $\delta_{\infty}(c)$ generate the same norm-closed ideal in $B$. Since unitarily equivalent elements generate the same ideal (Lemma 40), the hypothesis implies that $c$ and $\delta_{\infty}(c)$ likewise generate the same ideal in $\mathcal{M}(B) / B$, hence the same norm-closed ideal in $\mathcal{M}(B)$.

We shall show that the multipliers of $H:=\overline{\delta_{\infty}(c) B \delta_{\infty}(c)}$ contain an infinite family of equivalent, orthogonal projections summing to the identity (in the strict topology). Let $P_{i}=v_{i} v_{i}^{*}$. These projections are full in $\mathcal{M}(B)$, being equivalent to 1 , and since $H \cong T B T$ for some projection in $\mathcal{M}(B)$, we have that $\mathcal{M}(H)=T \mathcal{M}(B) T$. The $P_{i}$, being multipliers of $H$, are therefore projections in $T \mathcal{M}(B) T$. Thus the $P_{i}$ are full and sum to 1 in the multipliers of $H$ :

$$
\sum P_{i}=\sum T P_{i} T=1_{\mathcal{M}(H)} .
$$

Moreover, since the range projections for the $v_{i}$ are subordinate to $T$, a calculation shows that $T v_{i}^{*} T=T v_{i}^{*} v_{i} v_{i}^{*} T=T v_{i}^{*}$, and hence it follows that $T v_{i} T$ is still an isometry in $\mathcal{M}(H)$. Thus the $P_{i}$ are equivalent in the multipliers of $H$. Therefore, by the characterization of stability found by Hjemborg and Rørdam [10], the hereditary subalgebra $H$ is stable (and full in $\overline{B c B}$ ).

Corollary 13. Let $B$ be stable and $\sigma$-unital. If $C$ is a subalgebra of $\mathcal{M}(B)$, then $\delta_{\infty}(C)$ is a subalgebra having the property that $\overline{c B c}$ is stable and full in $\overline{B c B}$ for all positive $c$ in $\delta_{\infty}(C)$. 
Proof. A computation shows that $\delta_{\infty}$ is a $*$-homomorphism from $B$ into $\mathcal{M}(B)$, and hence for abstract reasons extends to a $*$-homomorphism from $\mathcal{M}(B)$ to $\mathcal{M}(B)$. Choose a positive element $c:=\delta_{\infty}(a) \in \delta_{\infty}(C)$. It follows from the above result that $H:=\overline{\delta_{\infty}(a) B \delta_{\infty}(a)}$ is stable and full in $\overline{B c B}$.

Lemma 14. Let $B$ be a stable and $\sigma$-unital $C^{*}$-algebra. Let $c$ be some element of $\mathcal{M}(B)^{+}$. An infinite repeat $\delta_{\infty}(c):=\sum_{1}^{\infty} v_{i} c v_{i}^{*}$ is full in $\mathcal{M}(\overline{B c B})$.

Proof. Since an ideal in a stable algebra is stable, $\overline{B c B}$ is stable, and since the inclusion of $\mathcal{M}(B)$ into $\mathcal{M}(\overline{B c B})$ is unital, the isometries defining $\delta_{\infty}$ are still isometries in $\mathcal{M}(\overline{B c B})$. Hence $\delta_{\infty}(c)$ is a diagonal element over $\overline{B c B}$. Lemmas 2.5 and 1.1 in Brown's paper [2] show that for a projection, $p \in B$, the tensor product $p \otimes 1$, which in our language is $\delta_{\infty}(p)$, will be full in $\mathcal{M}(B)$ if $p$ is full in $B$. Replacing $B$ by $\overline{B p B}$, we see that $\delta_{\infty}(p)$ is full in $\mathcal{M}(\overline{B p B})$. Since, as we have seen, $\overline{c B c}$ is equal to $T B T$, where $T$ is a projection, we have that $\delta_{\infty}(\overline{c B c})=\delta_{\infty}(T B T)$, and thus the general case follows from the case of projections.

A form of the converse is also true, and will be given later.

Next we have some lemmas on extensions.

Proposition 15. Let $\tau: A \longrightarrow \mathcal{M}(B) / B$ be a trivial essential extension. Then the extension algebra of this extension is isomorphic to $\hat{\tau}(A)+B$, where $\hat{\tau}: A \longrightarrow$ $\mathcal{M}(B)$ is any lifting of $\tau$ to a homomorphism into the multipliers. Moreover, if $\tau$ is unitarily equivalent by a multiplier unitary (modulo $B$ ) to its infinite repeat $\delta_{\infty}(\tau)$, then the extension algebra is isomorphic to $\delta_{\infty}(\hat{\tau}(A))+B$.

Proof. Consider the extension

$$
0 \longrightarrow B \longrightarrow \hat{\tau}(A)+B \stackrel{q}{\longrightarrow} A \longrightarrow 0
$$

where $q: \hat{\tau}(A)+B \longrightarrow A$ is defined by $q(M):=\hat{\tau}^{-1}(\pi(m))$, the map $\pi: \mathcal{M}(B) \longrightarrow$ $\mathcal{M}(B) / B$ being the standard quotient map. The Busby map of this extension is, by definition, $\tau_{M}(a):=\pi(c)$, where $c$ is any member of the inverse image of the singleton set $\{a\}$ under $q^{-1}$. It follows that $\tau_{M}(a)=\tau(a)$.

Since the extension algebra $C$ of an extension with Busby map $\tau: A \longrightarrow$ $\mathcal{M}(B) / B$ is (isomorphic to) a pullback

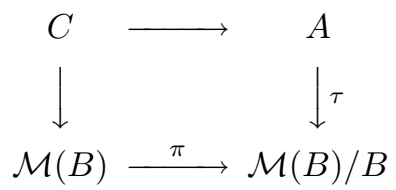

it follows (i.e. by the universal property) that two extensions with the same Busby map have isomorphic extension algebras. This proves the first statement.

For the second part, observe that if $\tau$ is unitarily equivalent to $\delta_{\infty}(\tau)$, then, in terms of the extension algebras, we have a commutative diagram

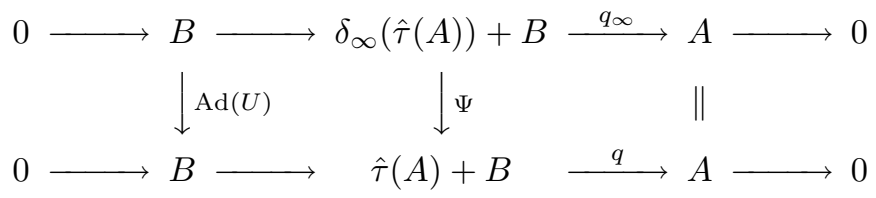

where $U \in \mathcal{M}(B)$ is the unitary implementing the equivalence, and $\Psi$ is an isomorphism coming from the universal property of pullbacks. 
2.1. Full extensions and the purely large condition. We can now clarify one of the questions asked in 6]: namely, if the purely large condition as defined there was equivalent to the apparently stronger form of the condition where the whole hereditary subalgebra was stable. We stress that the following theorem is for absorption without ideal-related restrictions; in other words, we are for the moment in the situation of [6], and are working with extensions that are full (i.e. essential extensions $\hat{\tau}: A \longrightarrow \mathcal{M}(B)$ having the property that $\hat{\tau}(a)$ is contained in a proper ideal of the multipliers if and only if the element $a$ is zero).

Moreover, we are just considering trivial extensions.

Theorem 16. Let $A$ and $B$ be $C^{*}$-algebras, with $B$ stable and $\sigma$-unital, and $A$ unital and separable. Let $0 \longrightarrow B \longrightarrow C \longrightarrow A \rightarrow 0$ be an essential unital trivial extension with weakly nuclear splitting homomorphism $s: A \longrightarrow C$. Then the following are equivalent:

i) The extension absorbs all trivial weakly nuclear extensions.

ii) The extension algebra has the approximation property that, for every $c \in C^{+}$ that is not zero in $C / B$ and every positive $b$ in $B$, there is an element $r \in B$ making the norm of $b-r c r^{*}$ arbitrarily small. Moreover, the element $r$ can be assumed to be of norm one if $b$ and $c$ are.

iii) The extension algebra has the property that, for every $c \in C^{+}$that is not zero in $C / B$, the hereditary subalgebra that it generates, $\overline{c B c}$, contains a stable subalgebra that is full in $B$.

iii') The extension algebra is isomorphic to $L+B$, where the algebra $L$ has the property that, for every nonzero $\ell \in L^{+}$, the hereditary subalgebra generated, $\overline{\ell B \ell}$, is stable and full in $B$.

Proof. The equivalence of i), ii), and iii) was shown in [6]. Now let us show that i) implies iii').

If $\hat{\tau}: A \longrightarrow \mathcal{M}(B)$ is (the lifting of) a trivial absorbing extension, then of course $\hat{\tau}$ is unitarily equivalent, modulo the canonical ideal $B$, to its infinite repeat. It then follows from Lemma12 that $H:=\overline{\delta_{\infty}(\hat{\tau}(a)) B \delta_{\infty}(\hat{\tau}(a))}$ is stable, and since the extension must necessarily be full in order to be absorbing (in the classical sense of [6]), the algebra $H$ is full in $B$.

By Lemma 15, we can identify the extension algebra of $\tau: A \longrightarrow \mathcal{M}(B) / B$ with $\delta_{\infty}(\hat{\tau}(a))+B$. Since both $\tau: A \longrightarrow \mathcal{M}(B)$ and $\delta_{\infty}: \mathcal{M}(B) \longrightarrow \mathcal{M}(B)$ are homomorphisms, $L:=\delta_{\infty}(\hat{\tau}(a))$ is a $C^{*}$-algebra.

Next, we show that iii') implies ii). Consider $\ell+b_{0}$, where $\ell \in L^{+}$and $b_{0} \in B$. Let $h \in B^{+}$be given, and suppose that $\ell$ and $h$ have norm equal to 1 . We shall find an element $r \in B$, with norm approximately equal to 1 , such that $r\left(\ell+b_{0}\right) r^{*}$ approximates $h$ arbitrarily closely in norm.

Since $\overline{\ell B \ell}$ is full in $B$, by Lemma 41, the element $\ell$ is full in $B$. Thus $h$ is in the ideal generated by $\ell$, and there are elements $g_{i} \in B$ such that

$$
\sum_{1}^{N} g_{i} \ell g_{i}^{*}
$$

approximates $h$. (The fact that the coefficients can be chosen symmetrically can be proven by a trick involving square roots in a matrix algebra; see [6, §7].) Recall that the multipliers of $\overline{\ell B \ell}$ are of the form $T \mathcal{M}(B) T$ for some multiplier projection $P \in \mathcal{M}(B)$, by Lemmas 10 and 11 . 
Choose $\epsilon>0$. Since $T b_{0} T$ is in $\overline{\ell B \ell}$ and $\overline{\ell B \ell}$ is stable, we can find orthogonal coisometries $u_{i}$ in $T \mathcal{M}(B) T$ with $u_{i} u_{j}^{*}=\delta_{i j}$ and

$$
\left\|u_{i} T b_{0} T\right\|<\frac{\epsilon}{\left\|g_{i}\right\|^{2} \cdot 2^{i}} .
$$

If we set $r_{0}:=\sum_{1}^{N} g_{i} \ell^{1 / 2} u_{i}$, then

$$
r_{0} T b_{0} T r_{0}^{*}=\sum_{1}^{N} g_{i} \ell^{1 / 2} u_{i} T b_{0} T u_{i}^{*} \ell^{1 / 2} g_{i}^{*}
$$

has norm less than $\epsilon$. Thus we see that this $r_{0}$ can be made approximately orthogonal to $b_{0}$. Furthermore,

$$
r_{0} r_{0}^{*}=\sum_{1}^{N} g_{i} \ell g_{i}^{*}
$$

approximates $h$, so of course $r_{0}$ has norm approximately equal to the square root of the norm of $h$, which has been assumed to be 1 . Also, since the $u_{i}$ are in $T \mathcal{M}(B) T$, we have that $r_{0} T=r_{0}$, and hence $r_{0} b_{0} r_{0}$ has arbitrarily small norm.

We have now proven, for all $\ell \in L^{+}$, that $h$ is approximated by $r_{0} r_{0}^{*}$ where $r_{0} \in \overline{B \ell}$, and $r_{0}$ is approximately orthogonal to $b_{0}$. In particular, we can find two functions $f$ and $g$ such that $f(\ell)$ is approximately equal to $\ell$, and $g(\ell) f(\ell)=g(\ell)$, by taking $g(\lambda)$ to be $(\lambda-1+\delta)^{+}$for some small number $\delta$, and taking $f$ to be equal to 1 on $(1-\delta, 1)$. The argument above gives us an $r_{1} \in \overline{B g(\ell)}$ such that $h$ is approximately equal to $r_{1} r_{1}^{*}$ and $r_{1}$ is approximately orthogonal to $b_{0}$. Then $r_{1}\left(\ell+b_{0}\right) r_{1}^{*}$ is approximately equal to $r_{1}\left(f(\ell)+b_{0}\right) r_{1}^{*}$, which in turn is approximately equal to $r_{1} f(\ell) r_{1}^{*}=r_{1} r_{1}^{*}$. This proves the claim.

Corollary 17. Let $A$ and $B$ be $C^{*}$-algebras, with $B$ stable and $\sigma$-unital, and $A$ unital and separable. Let $\tau: A \longrightarrow \mathcal{M}(B) / B$ be a trivial essential extension which is full in the sense that $\tau(a)$ is contained in a proper ideal of the corona if and only if a is zero. Then $\tau$ is absorbing in the nuclear sense if and only if it is an infinite repeat.

Remark. One might wonder if a result similar to the equivalence of i) and iii') in the theorem would hold for extensions which are not trivial. The difficulty is that the purely large part of the algebra, denoted above by $L$, is then apparently no longer an algebra. However, recall that the Weyl-von Neumann theorem of [6] shows that any absorbing extension contains any desired trivial extension, and in particular contains an infinite repeat:

Proposition 18 (6]). Let $A$ and $B$ be $C^{*}$-algebras, with $B$ stable and $\sigma$-unital, and $A$ unital and separable. Then, if $\psi$ is an absorbing, unital, and weakly nuclear extension, and $\tau$ is a trivial extension, there is an extension $\sigma$ such that $\psi=\tau \oplus \sigma$.

Combining the two results above, we see that a possibly not trivial extension $0 \longrightarrow B \longrightarrow C \longrightarrow A \longrightarrow 0$ will - if the various technical conditions given above are satisfied - be absorbing in the classical sense (that is, as defined in [6]) if and only if there is a projection $P$, associated with a generator of $O_{2}$ in $\mathcal{M}(B)$, such that $P C P=L+B$ where $L$ is a "purely large algebra" in the sense of part iii') of Theorem 16

In the remainder of the paper, we work in the context of ideal-related absorption. 


\section{A Weyl-Von NeUmann type theOREM}

The proof is a refinement of the one in the full case [6], and for the reader's convenience we will present the proof in the same sequence as in the full case. The theorem to be proven, modulo technicalities, is the following analytic form of a Weyl-von Neumann type theorem:

Theorem 19. Let $B$ be separable and stable. Let $C$ be a unital, factorizable and separable subalgebra of $\mathcal{M}(B)$ containing $B$. Let $\phi: C \longrightarrow \mathcal{M}(B)$ be a completely positive unital map, dominated by the identity. There exists a sequence $\left(v_{n}\right)$ such that, for each $c \in C, \phi(c)-v_{n} c v_{n}^{*}$ is in $B$, and goes to zero in norm as $n \longrightarrow \infty$.

The result is a corollary of the following remarkable proposition, which has probably been used, in one form or another, in all proofs of Weyl-von Neumann type theorems to date.

Proposition 20 (Kirchberg). Let $C$ be a unital separable subalgebra of $\mathcal{M}(B)$ containing $B$. Let $\phi: C \longrightarrow \mathcal{M}(B)$ be a completely positive unital map which is zero on $B$ and which has the property that, for every $b_{0}$ in $B$, the map

$$
b_{0} \phi b_{0}^{*}: C \longrightarrow B
$$

can be approximated on finite sets by maps of the form

$$
c \mapsto b c b^{*} \text {. }
$$

Then there exists a sequence $\left(v_{n}\right)$ such that, for each $c \in C, \phi(c)-v_{n} c v_{n}^{*}$ is in $B$, and goes to zero in norm as $n \longrightarrow \infty$.

A proof is given in [6].

The remarkable thing about this proposition is that the conclusion directly involves ideal structure, since it shows that $\phi: C \longrightarrow C$ is dominated by the identity map, modulo $B$, whereas the hypothesis is an approximation property, and of course the property of belonging to an ideal is not stable under small perturbations. One can now see the connection between the factorizability condition from Definition 7 and theorems of Weyl-von Neumann type.

Before proceeding, we make some remarks upon nuclearity. In the classical situation, one assumes that the extensions involved have weakly nuclear splitting maps, and this is then exploited in the proof of a Weyl-von Neumann type theorem by means of the following proposition or its equivalent:

Proposition 21. Let $C$ be a unital separable subalgebra of the multipliers $\mathcal{M}(B)$ and let $\phi: C \longrightarrow C$ be a completely positive map. The following are equivalent:

i) The map $\phi: C \longrightarrow C$ is nuclear.

ii) The map $\phi: C \longrightarrow C$ can be approximated over finite sets $\mathcal{C} \subset C$ by maps of the form

$$
c \mapsto \sum_{1}^{N} R_{i} \rho_{i}\left(F_{i}^{*} c F_{i}\right) R_{i}^{*},
$$

where the $R_{i}$ are row vectors of elements of $C$, the $F_{i}$ are row vectors of elements of $C$, and the $\rho_{i}$ are pure states of $C$, acting on $M_{n} \otimes C$ as $1 \otimes \rho_{i}$.

In this formulation, the above proposition is a slight generalization of lemma 9 in [6], where it is also pointed out that if the given map happens to be zero on the canonical ideal $B$, we can assume that the $\rho_{i}$ are zero on $B$. 
In the ideal-related context of interest, the above form of nuclearity is not the right tool, and we need to refine it to interact better with the hypothesis of idealdomination of one map by another. Let us say that $\phi: C \longrightarrow C$ is dominated by the identity in the nuclear sense if, upon finite sets, $\phi$ can be approximated by $c \mapsto \sum_{1}^{N} R_{i} \rho_{i}\left(F_{i}^{*} c F_{i}\right) R_{i}^{*}$ where the elements of $R_{i}$ are in $\overline{C g}$, for all $g$ with $\rho_{i}(g)=\|g\|=1$. In a more general form, we can say that:

Definition 22. Let $C$ be a $C^{*}$-algebra. The map $\phi: C \longrightarrow C$ is dominated by $\Psi: C \longrightarrow C$ in the nuclear sense if, upon finite sets, $\phi$ can be approximated by $c \mapsto \sum_{1}^{N} R_{i} \rho_{i}\left(F_{i}^{*} c F_{i}\right) R_{i}^{*}$ where the elements of $R_{i}$ are in $\overline{C \Psi(g)}$, for all $g$ with $\rho_{i}(g)=\|g\|=1$.

There appears to be a trade-off between placing conditions on the algebra in a theorem of Weyl-von Neumann type and placing conditions on the map, in this case meaning the $C S$ condition on the algebra versus the nuclearity-related conditions on the map. The factorizability condition, on the other hand, is not negotiable, as it is clearly a necessary condition for any absorption result to hold. Let us now see what the $C S$ condition, factorizability condition, and ideal-domination condition can be used for. At a later stage, we will also need the local fullness condition!

We begin with some lemmas, the first of which lets us lift the $C S$ property from the quotient algebra.

Lemma 23. Let $C$ be a separable algebra containing an ideal $B$. If $\phi: C \longrightarrow \mathbb{C}$ is a pure state on $C$ that is zero on $B$ and if $C / B$ has the $C S$ property, then the hereditary kernel in $C$ of $\phi$ contains a strictly positive element $h$ with $s p_{C} h=$ $[0,\|h\|]$.

Proof. Let $N$ be the kernel of $c \mapsto \phi\left(c^{*} c\right)$. Since $N$ is a maximal left ideal of $C$ and $B$ is in $N$, it follows that $N / B$ is a maximal left ideal of $C / B$, so that $\phi$ is a pure state on $C / B$.

Let $h$ be a strictly positive element of continuous spectrum for the hereditary kernel $\frac{N \cap N^{*}}{B}$ of $\phi: C / B \longrightarrow \mathbb{C}$. We can lift $h$ to a positive element $\tilde{h}$ of $C$. This lifting need not be strictly positive, but, from the next lemma, it follows that $\tilde{h}+b$, where $b$ is strictly positive for $B$, will itself be strictly positive in $C$. It is easier to be invertible in $\mathrm{C} / \mathrm{B}$ than it is in $C$, so the Hilbert space spectrum of $\tilde{h}+b$ contains that of $h$. If we consider the function $f(\lambda)$ that is equal to $\lambda$ when $0 \leq \lambda \leq\|h\|_{C / B}$ and is equal to $\|h\|_{C / B}$ otherwise, then $f(\tilde{h}+b)$ will be a strictly positive lifting of $h$ with spectrum $[0,\|h\|]$.

Lemma 24. If $I$ is an ideal in a $C^{*}$-algebra $C$, and $j \in I^{+}, c \in C^{+}$are such that $j$ is strictly positive in $I$ and $c+B$ is strictly positive in $C / B$, then $j+c$ is strictly positive in the algebra $C$.

Proof. Let $g \in C^{+}$and $\epsilon>0$ be arbitrary. Let $\pi: C \longrightarrow C / B$ be the usual canonical quotient map. By Cohen's theorem [4] applied to $C / I$, we have that $\pi(g)=f(\pi(c)) q\left(f(\pi(c))\right.$ for some $C_{0}(0, \infty)$ function $f$ and some element $q$ of $C / B$.

Thus we have the inequality

$$
\pi(g) \leq\|q\| f(\pi c))^{2},
$$

and for geometrical reasons, there is a constant $C_{1}$ such that

$$
\|q\| f(\lambda)^{2} \leq C_{1} \lambda+\epsilon
$$


for all positive real $\lambda$. Therefore,

$$
\pi(g) \leq C_{1} \pi(c)+\epsilon
$$

in the unitization $(C / I)^{\sim}$.

Next, consider the positive part of the expression $g-\left(C_{1} c+\epsilon\right)$. We see that $\left(\left(g-\left(C_{1} c+\epsilon\right)\right)^{+}\right.$is in $I$, and since $j$ is strictly positive in $I$, an argument similar to the above shows that there is a constant $C_{2}$ such that

$$
\left(g-\left(C_{1} c+\epsilon\right)\right)^{+} \leq C_{2} j+\epsilon .
$$

It follows that $g \leq C_{1} c+C_{2} j+2 \epsilon$ and hence that $g \leq C_{3}(c+j)+2 \epsilon$ for some constant $C_{3}$. Since $g$ and $\epsilon$ were arbitrary, it follows that there cannot be a state $\phi$ such that $\phi(c+j)=0$.

The next lemma is a modification of a result from [1], and has a counterpart in the nonunital case.

Lemma 25 (Excision of states lemma). Let $C$ be a separable unital $C^{*}$-subalgebra of $\mathcal{M}(B)$ with the quotient having the $C S$ property, and let $\rho$ be a pure state of $C$ that is zero on $B$. There is a positive element $g \in C$, of unit norm, such that

i) the state applied to $g$ gives $\rho(g)=1$,

ii) $(1-g)^{1 / n}$ is an approximate unit for $B$,

iii) the spectrum $s p_{C} g$ of $g$ is $[0,1]$, and

iv) $\lim _{n \longrightarrow \infty}\left\|g^{n}(c-\rho(c)) g^{n}\right\|=0$ for each $c$ in $C$.

Proof. Since $\rho$ is pure, $C$ can be decomposed as

$$
C=\mathbb{C}+N+N^{*}
$$

where $N$ is the kernel of $c \mapsto \rho\left(c^{*} c\right)$. Since $N$ is a separable one-sided ideal, $H:=$ $N \cap N^{*}$ is a hereditary subalgebra of $C$ that contains $B$. Let $\ell$ be a strictly positive element of $N \cap N^{*}$, with norm equal to 1 in $H / B$. By the proof of Lemma 23. we can arrange that this element not only is strictly positive in $H$, but also has spectrum $[0,1]$. Let the desired element $g$ be $1-\ell$. Then, as $B$ is contained in $H$, we have that $(1-g)^{1 / n}$ is an approximate unit for $B$.

If $c$ is any element of the kernel of $\rho$, then by the strict positivity of $\ell$ in $H$ the element $c$ is approximately of the form $\ell x+y \ell$ for some $x$ and $y$. Since $\ell(1-\ell)^{n}$ goes to zero in norm, $g^{n} c g^{n}$ goes to zero in norm. Finally, since $\ell$ is in the kernel of the state, clearly $\rho(g)$ is equal to 1 .

We can now prove the counterpart of Corollary 9 of [6] in our setting.

Lemma 26. Let $C$ be factorizable with respect to $B$, and have the $C S$ property. Given a completely positive unital map $\phi: C / B \longrightarrow B$ of the identity-dominated nuclear form $\phi(c)=R \rho\left(F^{*} c F\right) R^{*}$ where $\rho$ is a pure state that is zero on $B, R$ is a row vector over the ideal determined by the support of the map, and $F$ is a row vector over $C$, the map can be approximated over a given compact set by $c \mapsto z c z^{*}$.

Proof. Let $\mathcal{C}$ be the finite set over which we want the approximation. Use the above excision of states lemma to find an element, $g \in C$, with unit norm and with $g^{2 n} \rho\left(F^{*} \mathcal{C} F\right)$ approximately equal to $g^{n} F^{*} \mathcal{C} F g^{n}$, elementwise, for some exponent $n$. Using an approximate unit, we can find an element $h$ in the hereditary subalgebra generated by $g$ such that $R h$ is approximately equal to $R$. 
Recall that the factorizability property (Definition 7, see also Corollary [5) gives that if $h$ is in the hereditary subalgebra generated by some multiplier element $g^{n}$, we have - granting the isospectrality condition, which comes from the CS property that $h$ is approximately equal to $r g^{n} r^{*}$. Thus, $R$ is approximately equal to $R r g^{n} r^{*}$

Now observe that since $R$ is a row vector of elements and $F^{*}$ is a row vector of elements, $z:=R r g^{n} r F^{*}$ is in $B$, and the above approximations show that $z c z^{*}$ is approximately equal to $\phi(c)$ for all $c$ in the given finite set. We then obtain the claimed approximation.

In the above result, one can perturb $z$ to a co-isometry.

Corollary 27. Let $C$ be a unital, factorizable, separable subalgebra of $\mathcal{M}(B)$ containing $B$. A completely positive map from $C$ to $B$ which is zero on $B$ and is dominated by the identity in the nuclear sense can be approximated on finite sets by maps of the form

$$
c \mapsto b c b^{*} .
$$

Proof. We may as well suppose that the elements in the finite set are positive. By hypothesis, the map can be approximated over finite sets by sums of maps of the form $c \mapsto R \rho\left(F^{*} c F\right) R^{*}$, with $R$ and $F$ as in the previous lemma. By a trick due to Arveson - and also used in lemma 10 of [6] — we can in effect reduce the case of a finite sum of maps to the case of a single such map. We shall only consider the case of a sum of two maps, since the general case involves no additional ideas. Let us suppose that the given map is approximated over the given finite set $\mathcal{C}$ by

$$
c \mapsto R \rho_{1}\left(F_{1}^{*} c F_{1}\right) R_{1}^{*}+R_{2} \rho_{2}\left(F_{2}^{*} c F_{2}\right) R_{2}^{*} .
$$

Then, use Lemma 26 to approximate the first term by $c \mapsto z_{1} c z_{1}^{*}$. Next, apply the same lemma to the second term, with respect to the larger finite set

$$
\mathcal{C} \cup\left\{c z_{1}^{*} z_{1} c: c \in \mathcal{C}\right\}
$$

obtaining a map $c \mapsto z_{2} c z_{2}^{*}$ that is approximately zero on all elements of the above finite set that are in $B$. In particular, $z_{2} c z_{1}^{*} z_{1} c z_{2}^{*}$ is approximately zero, and hence by the properties of the $C^{*}$-norm, $z_{1} c z_{2}^{*}$ is approximately zero for all $c \in \mathcal{C}$.

Thus the given map is approximated over $\mathcal{C}$ by $c \mapsto\left(z_{1}+z_{2}\right) c\left(z_{1}+z_{2}\right)^{*}$, where the cross terms are negligible by the above construction.

Further, we have the following corollary:

Corollary 28. Let $C \subset \mathcal{M}(B)$ be unital, separable, and factorizable. Suppose also that $B \subset C$. Let $\tau: C \longrightarrow \mathcal{M}(B)$ be a unital completely positive map which is zero on $B$ and is dominated by the identity in the nuclear sense. Then $\tau$ is approximately unitarily equivalent to $c \mapsto p c p$ for some projection $p \in \mathcal{M}(B)$.

Proof. We follow Kasparov's version [7] of Arveson's proof. The above Corollary 28 establishes the conditions needed to apply Proposition 20. Thus we can approximate $\tau$ in the desired sense by $c \mapsto v^{*} c v$ for some $v \in \mathcal{M}(B)$. Since $\tau$ is unital, we can take $v$ to be an isometry and define a projection $p$ by $p=v v^{*}$. Since $B$ is stable, the multipliers $\mathcal{M}(B)$ are isomorphic to the bounded adjointable operators $\mathcal{L}\left(\mathcal{H}_{B}\right)$ on the standard Hilbert module $\mathcal{H}_{B}$, and in particular, the two maps

$$
\tau: C \longrightarrow \mathcal{L}\left(\mathcal{H}_{B}\right)
$$


and

$$
v \tau v^{*}: C \longrightarrow \mathcal{L}\left(p \mathcal{H}_{B}\right)
$$

are unitarily equivalent in the multipliers, since $v$ is an isometry.

Clearly, $c \mapsto p c p$ will approximate $c \mapsto v \tau(c) v^{*}$, so we are done.

Let us say that maps for which domination and domination in the nuclear sense coincide are $I R$-nuclear. Then we can rewrite this corollary more briefly as:

Corollary 29. Let $C \subset \mathcal{M}(B)$ be unital, separable, and factorizable. Suppose also that $B \subset C$. Let $\tau: C \longrightarrow \mathcal{M}(B)$ be a unital completely positive map which is zero on $B$, is IR-nuclear, and is dominated by the identity. Then $\tau$ is approximately unitarily equivalent to $c \mapsto p c p$ for some projection $p \in \mathcal{M}(B)$.

3.1. Repeats and ideal-related absorption. We are now prepared to, using some of the results from the previous section as lemmas, convert the Weyl-von Neumann theorem proven earlier into a result about ideal-related absorption of extensions. The main new problem we face, which has no counterpart in the case of full extensions, is that apparently the process of taking the infinite repeat of an extension might not preserve ideal structure. It turns out that the extensions we deal with need to be locally full, in a sense suggested by the next lemma.

Lemma 30. Let $B$ be a stable $C^{*}$-algebra. If $c$ is full in $\frac{\mathcal{M}(\overline{B c B})}{\overline{B c B}}$, then $\delta_{\infty}(c)$ and $c$ generate the same ideal in $\mathcal{M}(B)$.

Proof. It is easy to show that the multiplier ideal generated by $c$ is contained in the ideal generated by $\delta_{\infty}(c)$. We now show that equality holds.

Writing $\delta_{\infty}$ explicitly in terms of generators $\left(v_{i}\right)$ of a copy of $O_{\infty}$, we notice that for all $b_{1}, b_{2} \in B$, the sum

$$
b_{1} \delta_{\infty}(c) b_{2}=\sum_{1}^{\infty} b_{1} v_{i} c v_{i}^{*} b_{2}
$$

converges in norm. Since the $v_{i}$ are isometries, it follows that (as in the proof of Proposition 12

$$
\overline{B \delta_{\infty}(c) B}=\overline{B c B} .
$$

Hence, the two elements $c$ and $\delta_{\infty}(c)$ generate the same ideal in $B$, and as $c$ is full in the local corona,

$$
\frac{\mathcal{M}(\overline{B c B})}{\overline{B c B}}=\frac{\mathcal{M}\left(\overline{B \delta_{\infty}(c) B}\right)}{\overline{B \delta_{\infty}(c) B}},
$$

we have that they generate the same ideal in $\mathcal{M}(\overline{B c B})$.

Next, choosing $\epsilon>0$, we have

$$
\left\|1-\sum_{1}^{n} g_{i} c g_{i}^{*}\right\|<\epsilon
$$

in $\mathcal{M}(\overline{B c B})$. Choosing a positive element $h$ in the unit ball of $\overline{\mathcal{M}(B) \delta_{\infty}(c) \mathcal{M}(B)}$, we have that

$$
\left\|h-\sum_{1}^{n}\left(h^{1 / 2} g_{i} c^{1 / 3}\right) c^{1 / 3}\left(c^{1 / 3} g_{i}^{*} h^{1 / 2}\right)\right\|<\epsilon
$$

where the $g_{i}$ are in $\mathcal{M}(\overline{B c B})$, not in $\mathcal{M}(B)$. However, it follows from our main lemma on local multipliers (Lemma [38) that the expressions in parentheses are actually in the multipliers of $B$, and therefore $h$ is in the multiplier ideal generated 
by $c$. We have now shown that the positive cone of the multiplier ideal generated by $c$ is the same as the positive cone of the multiplier ideal generated by $\delta_{\infty}(c)$.

Lemmas 14 and 30 together show that local fullness, in the sense of the above lemma, is a necessary and sufficient condition for $c$ and $\delta_{\infty}(c)$ to generate the same multiplier ideal.

Definition 31. Recall that an extension is locally full if, for every positive element $c$ of the extension algebra, regarded as a subalgebra of the multipliers, $c$ is full in $\frac{\mathcal{M}(\overline{B c B})}{\overline{B c B}}$.

Proposition 32. If $\tau$ is a trivial extension with infinite repeat $\tau_{\infty}$, and if $\tau$ is dominated by a locally full extension $\sigma$, then $\tau_{\infty}$ is dominated by $\sigma$.

Proof. This is almost a corollary of the proof of the last lemma. Choosing some positive element $a$ in the domain of $\tau$, it is clear that $\hat{\tau}(a)$ is in the ideal generated by $\hat{\tau}_{\infty}(a)$.

Since $\hat{\tau}(a)$ is in the multiplier ideal generated by $\hat{\sigma}(a)$, we have, much as before, that

$$
\tilde{\tau}(a)-\sum_{1}^{n}\left(\tilde{\tau}(a)^{1 / 2} g_{i} c^{1 / 3}\right) c^{1 / 3}\left(c^{1 / 3} g_{i}^{*} \tilde{\tau}(a)^{1 / 2}\right)
$$

where the expressions in parentheses are in $\mathcal{M}(B)$, by Lemma 38 .

Even though $\sigma$ is not a trivial extension, we can still apply $\delta_{\infty}: \mathcal{M}(B) \longrightarrow \mathcal{M}(B)$ to elements of the image of $\hat{\sigma}: A \longrightarrow \mathcal{M}(B)$. Writing the above sum concisely as

$$
\tilde{\tau}(a) \approx \sum_{1}^{n} m_{j} \tilde{\sigma}(a)^{1 / 3} m_{j}^{*}
$$

we can apply the previously defined map $\delta_{\infty}$ to both sides. Since $\delta_{\infty}$ is a homomorphism, it is norm-decreasing and does not increase the error in the approximation. Thus we obtain an approximation of $\hat{\tau}(a)$ by

$$
\sum_{1}^{n} m_{j} \delta_{\infty}(\hat{\sigma}(a))^{1 / 3} m_{j}^{*}
$$

and by the local fullness of $\hat{\sigma}$, the element $\delta_{\infty}\left(\hat{\sigma}(a)^{1 / 3}\right)$ is in the ideal generated by $\hat{\sigma}(a)$ in $\mathcal{M}(B)$, so we are done.

One corollary of the proposition is:

Corollary 33 (The sandwich theorem). If $\tau$ is a trivial extension, dominated by a locally full extension $\sigma$, there exists a trivial locally full extension that is dominated by $\sigma$ and dominates $\tau$.

We can now combine the above result with Corollary[29] to obtain the following:

Theorem 34. Let $\tau$ be an essential, IR-nuclear, unital extension of a $\sigma$-unital stable $C^{*}$-algebra, $B$, by a unital separable $C^{*}$-algebra A. If $\tau$ is factorizable and locally full, then it absorbs every trivial IR-nuclear extension that it dominates.

Proof. Let the trivial extension be $\hat{\pi}: A \longrightarrow \mathcal{M}(B)$, with infinite repeat denoted by $\hat{\pi}_{\infty}: A \longrightarrow \mathcal{M}(B)$. The infinite repeat is both trivial and locally full, and, by Proposition [32, it is dominated by $\hat{\tau}: A \longrightarrow \mathcal{M}(B)$. To show that $\hat{\tau}$ absorbs $\hat{\pi}$, it is sufficient to show that $\hat{\tau}$ is the BDF sum of some other extension with $\hat{\pi}_{\infty}$. 
Note that since both $\hat{\tau}: A \longrightarrow \mathcal{M}(B)$ and $\hat{\pi}_{\infty}: A \longrightarrow \mathcal{M}(B)$ are injective, if $c$ is a positive element of the image of $\hat{\tau}$, we have that the multiplier ideal generated by $\hat{\pi}_{\infty}\left(\hat{\tau}^{-1}(c)\right)$ is contained in that generated by $c$; in other words,

$$
\begin{aligned}
I\left(\hat{\pi}_{\infty}\left(\hat{\tau}^{-1}(c)\right)\right) & \subseteq I\left(\hat{\tau}\left(\hat{\tau}^{-1}(c)\right)\right) \\
& =I(c) .
\end{aligned}
$$

Hence, the map $\phi: C \longrightarrow \mathcal{M}(B)$ that we apply the Weyl-von Neumann theorem to is dominated by the identity map, and by the corollary to the absorption theorem (Corollary [28), there is a projection $p$, equivalent to 1 , such that $p \hat{\tau} p=\hat{\pi}_{\infty}$, with approximate unitary equivalence as the form of equivalence of the extensions. Replacing $\hat{\pi}_{\infty}$ in this argument by $\hat{\pi}_{\infty} \oplus \hat{\pi}_{\infty}$, we have a projection, still denoted $p$, such that $p \hat{\tau} p=\hat{\pi}_{\infty} \oplus \hat{p} i_{\infty}$. Since we are dealing with homomorphisms into the corona, the projection $p$ commutes with the image of $\tau$ (modulo $B$ ), and we can write

$$
p \hat{\tau}=\hat{\pi}_{\infty} \oplus \hat{\pi}_{\infty} .
$$

If we consider the projections associated with the copy of $\mathrm{O}_{2}$ implementing the addition of the two extensions on the right, we get two orthogonal projections $e_{i}$, each equivalent to 1 , with $p=e_{1}+e_{2}$ and $e_{i} \tau=\pi_{\infty}$. Hence

$$
\begin{aligned}
\tau= & (1-p) \tau+e_{1} \tau+e_{2} \tau \\
& =\left(1-p+e_{1}\right) \tau+e_{2} \tau .
\end{aligned}
$$

We claim that $\left(1-p+e_{1}\right)$ and $e_{2}$ are orthogonal projections that are equivalent to 1. In this case we would have that

$$
\begin{gathered}
\hat{\tau}=s_{1} s_{1}^{*} \hat{\tau} s_{1} s_{1}^{*}+s_{2} s_{2}^{*} \hat{\tau} s_{2} s_{2}^{*} \\
=s_{1} \hat{\sigma} s_{1}^{*}+s_{2} \hat{\pi}_{\infty} s_{2}^{*},
\end{gathered}
$$

where $s_{1}$ and $s_{2}$ are isometries defined by $s_{1} s_{1}^{*}=\left(1-p+e_{1}\right)$ and $s_{2} s_{2}^{*}=e_{2}$, and the extension $\sigma$ is defined by $s_{1}^{*} \hat{\tau} s_{1}$. Since $s_{1}$ and $s_{2}$ generate a copy of $O_{2}$, we will have that $\hat{\tau}$ is the BDF sum of $\hat{\pi}_{\infty}$ and $\hat{\sigma}$. To complete the proof we need only show that $e_{2}$ and $\left(1-p+e_{1}\right)$ have the properties claimed. The first of the following lemmas shows that $e_{i}$ is orthogonal to $1-p$, and the second shows that $\left(1-p+e_{1}\right)$ is equivalent to 1 (since $e_{1}$ is).

Lemma 35. If $a$ and $b$ are projections with $\|a+b\| \leq 1$, they are orthogonal.

The proof is omitted.

Lemma 36. If $e$ and $f$ are projections in $\mathcal{M}(B \otimes \mathcal{K})$ with $e \leq f$ and e equivalent to 1 , then $f$ is equivalent to 1 .

This lemma is proven by Mingo [11].

The proof of the theorem is now complete.

The next theorem shows how to obtain absorbing extensions by piecing them together from extensions of smaller subalgebras.

Theorem 37. Let $\tau$ be an essential, unital, IR-nuclear extension of a $\sigma$-unital stable algebra $B$ by a unital separable algebra $A$. Then the following are equivalent:

i) The extension $\hat{\tau}: A \longrightarrow \mathcal{M}(B)$ is locally full and factorizable.

ii) The extension is absorbing in the ideal-related sense (with respect to approximate unitary equivalence). 
iii) The restrictions $\hat{\tau}_{a}: C^{*}(a)^{\sim} \longrightarrow \mathcal{M}(B)$ are absorbing in the ideal-related sense (with respect to approximate unitary equivalence).

Remark. This theorem demonstrates that absorption is in some sense a local property.

Proof. That i) implies ii) follows from Theorem 34. It is clear that ii) implies iii), and we next show that iii) implies i).

Observe that

$$
\tau_{a}: \frac{C^{*}(a)^{\sim}}{B} \longrightarrow \frac{\mathcal{M}(B)}{B}
$$

is an injective nuclear homomorphism on an abelian $C^{*}$-algebra. Let $c$ be the image of $\hat{\tau}(a)$ in the multipliers, for some positive element $a$ with connected spectrum, and $h$ be a positive element of the ideal generated by $c$, so that $h$ and $c$ satisfy the hypotheses of the factorizability condition. Since $c$ has spectrum $[0,\|c\|]$ in the corona (and hence in the multipliers), we can, as in Proposition 4, consider

$$
\begin{aligned}
\hat{\tau}_{a}: C^{*}(a) / B & \longrightarrow \mathcal{M}(B) \\
f(a) & \mapsto f(c)
\end{aligned}
$$

and

$$
\begin{aligned}
\hat{\Phi}: C^{*}(a) / B & \longrightarrow \mathcal{M}(B) \\
f(a) & \mapsto f\left(c^{\prime}\right),
\end{aligned}
$$

where $c^{\prime}$ is some element isospectral to $c$, and in the ideal generated by $c$. Since both $a$ and $c^{\prime}$ have spectra $[0,\|c\|]$, the map $\hat{\Phi}$ is injective, and hence essential. Moreover, $\Phi$ is trivial, so we have that $\hat{\tau}_{a}$ absorbs $\hat{\Phi}$. Hence $\hat{\tau}_{a} \oplus \hat{\Phi}$ is approximately unitarily equivalent to $\hat{\Phi}$, and cutting down by a projection we have $f\left(c^{\prime}\right)=r f(c) r^{*}+b$ for some $b$ of arbitrarily small norm.

Since $h$ has a square root in $\overline{c \mathcal{M}(B) c}$, we can use Cohen's theorem to factorize $h^{1 / 2}$ as $f(c) h^{\prime} f(c)$ for some $h^{\prime}$ with norm approximately equal to one, and some $f(c)$ with norm equal to one. Noting that $c$ and $f(c)^{2}$ are isospectral and that $f(c)^{2}$ is in the ideal generated by $c$, we can take $c^{\prime}$ in the previous argument to be $f(c)^{2}$. Thus, $h$ is approximated by $f(c) h^{\prime} r c r^{*} h^{\prime} f(c)$. This establishes the factorization condition. As has been pointed out before, the definition of absorption as it is usually applied implicitly contains the requirement to absorb infinite repeats of a subordinate extension, and recalling that infinite repeats are locally full (Lemma 14), we see that $\tau$ must be locally full.

\section{Technical lemmas}

Lemma 38. Let $B$ be $\sigma$-unital. If $c$ is an element of a multiplier algebra $\mathcal{M}(B)$ and $d$ is in the multiplier ideal generated by $c$, then $\overline{d \mathcal{M}(\overline{B c B}) d^{*}}$ is contained in the multipliers of $B$.

Proof. We must show that an element of the given form is a well-defined two-sided multiplier of $B$. Consider first of all the cases where the element is of the form $\mathrm{cmc}$ with $m$ in the multipliers of $\overline{B c B}$. If $b$ is in $B$, we define an action of $c m c$ on $b$ by approximating $c m c b$ by $c m(h c b)$, where $h$ comes from an approximate unit for $B$ and where $m$ can now be applied on the right. The general case can be obtained by also approximating $d$ by a finite sum $\sum m_{k} c m_{k}^{\prime}$. 
Lemma 39. The ideal generated by a positive element $x$ is the same as the ideal generated by $f(x)$, where $f \in C_{0}((0,1])$ is a function that is invertible under composition.

Proof. By "rounding off" the function $f(\lambda) / \lambda$, we can find a not necessarily bounded sequence of $C_{0}$ functions $g_{n}$ such that $x g_{n}(x)$ goes to $f(x)$ in norm. Therefore, $f(x)$ is contained in the ideal generated by $x$. Since $f$ is invertible under composition, the reverse inclusion also holds.

Lemma 40. The ideal generated by $x^{*} x$ is the same as the ideal generated by $x x^{*}$.

Proof. Let $I\left(x^{*} x\right)$ denote the ideal generated by $x^{*} x$. Clearly $I\left(x^{*} x\right)$ contains the ideal $I\left(\left(x x^{*}\right)^{2}\right)$, but by the above lemma it follows that $I\left(x^{*} x\right)$ contains $I\left(x x^{*}\right)$. The reverse inclusion follows by replacing $x$ by $x^{*}$.

Lemma 41. Let $B$ be $\sigma$-unital. The ideal generated by $\overline{c B c}$ is the same as the ideal generated by the positive element $c \in \mathcal{M}(B)$.

Proof. Take a strictly positive element $b$ in $B$. By the above lemma, $b^{1 / 2} c b^{1 / 2}$ and $c^{1 / 2} b c^{1 / 2}$ generate the same ideal in $B$. However, $b^{1 / 2} c b^{1 / 2}$ generates the same ideal as $c$, but $c^{1 / 2} b c^{1 / 2}$ is strictly positive in $\overline{c B c}$.

\section{Final comments}

i) The standard equivalence relation for extensions is unitary equivalence modulo the canonical ideal: i.e. $\hat{\tau}(a)=U \hat{\sigma}(a) U^{*}+b$, with $b$ depending on $a$. The stronger notion of approximate unitary equivalence, used here and sometimes used in the classification theory for $C^{*}$-algebras, is obtained if we also require that the norm of $b$ can be made arbitrarily small (pointwise with respect to $a \in A$ ) by choosing $U$ suitably. We are led to ask if the resulting equivalence classes within the set of ideal-related purely large extensions are in fact the same. This appears to be likely, since absorption theorems generally give approximate unitary equivalence.

ii) It appears that infinite repeats do not behave as well in the ideal-related situation as they do in the classical situation. In particular, is it true that infinite repeats must necessarily be absorbing in the ideal-related sense? This would seem plausible, but difficult to prove.

The definition of absorption, as it is usually applied, is infinite absorption. In other words, if $\tau$ absorbs $\sigma$, then $\tau$ absorbs $\sigma \oplus \sigma \oplus \sigma \oplus \cdots$. One could equally well study finite absorption, where we just drop this condition, and presumably one would then be led to a theory of absorbing extensions that are not locally full. Infinite repeats would have no natural place in such a theory.

iii) An inspection of the proofs of the theorems shows that the stability of $B$ is used primarily in defining the sum of extensions: first, in connection with the construction of infinite repeats, using a copy of $O_{\infty}$ in the multipliers; and second, in using a unital copy of $\mathrm{O}_{2}$ to define pairwise addition of extensions. Since $\mathrm{O}_{2}$ contains a copy of $O_{\infty}$, it seems likely that stability could be replaced by the existence of a copy of $\mathrm{O}_{2}$ in the multipliers.

iv) Consider the set of all absorbing (in the ideal-related sense), nuclear, essential, and separable extensions of two given $C^{*}$-algebras. Then, a natural question is to ask what algebraic structure can be assigned to this set. The BDF construction shows that the set is a semigroup, and clearly the semigroup can be partitioned 
into subsemigroups, with each subsemigroup corresponding to an ideal class (i.e. the map induced by a Busby map on the lattice of ideals). Moreover, one of these ideal classes (the class of full extensions) is actually the group $K K^{1}(A, B)$.

In this paper we have shown that trivial extensions satisfying appropriate hypotheses are algebraically trivial within each ideal class of extensions. Among open questions, we mention the following: do there exist trivial extensions in each ideal class?

\section{ACKNOWLEDGEMENTS}

We thank George Elliott for valuable discussions.

\section{REFERENCES}

1. C.A. Akemann, J. Anderson, and G.K. Pedersen. Excising states of $C^{*}$-algebras, Canad. J. Math. 38 (1986). MR 88d:46107

2. L. Brown. Stable isomorphism of hereditary subalgebras of $C^{*}$-algebras, Pacific J. Math $\mathbf{7 1}$ (1977), 335-384. MR 56:12894

3. L. Brown, R. Douglas, P. Fillmore, Unitary equivalence modulo the compact operators and extensions of $C^{*}$-algebras, Lecture Notes in Math. 345 (1973), 58-128. MR 52:1378

4. P.J. Cohen, Factorization in Group Algebras, Duke Math. J. 26 (1959), 199-205. MR 21:3729

5. G.A. Elliott, Derivations of matroid $C^{*}$-algebras, II, Ann. of Math 100 (1974), 407-422. MR 50:5485

6. G.A. Elliott, D. Kucerovsky, An abstract Brown-Douglas-Fillmore absorption theorem, Pacific J. of Math. 198 (2001), 385-409. MR 2002i:46052

7. G.G. Kasparov, Hilbert $C^{*}$-modules: theorems of Stinespring and Voiculescu, J. Op. Th. 4 (1980), 133-150. MR 82b:46074

8. E. Kirchberg, The classification of purely infinite $C^{*}$-algebras using Kasparov's theory Ktheory, preprint, 1995.

9. E. Kirchberg and M. Rørdam, Nonsimple purely infinite $C^{*}$-algebras, preprint, 1999. MR 2001k:46088

10. J. Hjelmborg and M. Rørdam, Stablility of $C^{*}$-algebras, JFA, 1998. MR 99g:46079

11. J.A. Mingo, K-theory and multipliers of stable $C^{*}$-algebras, Tr. AMS. 299 (1987), 397-411. MR 88f:46136

12. G.K. Pedersen, A strict version of the non-commutative Urysohn lemma, Proc. AMS. 125 (1997), 2657-2660. MR 97j:46061

13. M. Rørdam, Ideals in the multiplier algebra of a stable $C^{*}$-algebra, J. Op. Th. 25 (1991), 283-298. MR 94c:46118

14. S. Zhang, Certain $C^{*}$-algebras with real rank zero and their corona and multiplier algebras, Part I, Pacific J. Math. 155 (1992), 169-197. MR 94i:46093

Department of Mathematics and Statistics, University of New Brunswick-Fredericton, Fredericton, New Brunswick, Canada E3B 5A3

E-mail address: dkucerov@unb.ca 